\title{
Evaporation and coarsening dynamics with open boundaries
}

\author{
A. M. Lacasta \\ Departament de Física Aplicada, Universitat Politècnica de Catalunya, Avinguda Dr. Gregorio Marañón, 50, E-08028 Barcelona, Spain \\ F. Sagués \\ Departament de Química Física, Universitat Barcelona, Avinguda Diagonal 647, E-08028 Barcelona, Spain \\ J. M. Sancho \\ Departament d'Estructura i Constituents de la Matèria, Universitat de Barcelona, Avinguda Diagonal 647, E-08028 Barcelona, Spain \\ I. M. Sokolov \\ Theoretishe Polymerphysik, Universität Freiburg, Hermann-Herder-Straße 3, D-79104 Freiburg i. Br., Germany
}

(Received 14 August 1998)

\begin{abstract}
We present a study of the evaporation dynamics of a substance undergoing a coarsening process. The system is modeled by the Cahn-Hilliard equation with absorbing boundaries. We have found that the dynamics, although of a diffusive nature, is much slower than the usual one without coarsening. Analytical and simulation results are in reasonable agreement. [S1063-651X(99)05701-3]
\end{abstract}

PACS number(s): 05.70.Ln, 83.70.Hq, 64.70.Fx

Phase separation in binary systems is an interesting example of pattern formation in nonequilibrium systems [1-8]. The system, placed in an unstable state, evolves spontaneously towards the equilibrium by generating domains rich in each of the two components. When the volume fraction of one of the components is sufficiently small, the domains of the minority phase coarsen to form circular domains (droplets) immersed in the bulk of the majority phase. In its late stages, this process can be described by Lifshitz-Slyosov theory [3], which assumes that the larger droplets grow at the cost of smaller ones, which are thermodynamically less stable due to their higher surface energy. From a theoretical and practical point of view, phase separation processes are usually modeled by time-dependent Ginzburg-Landau equations for the local concentration, with a conserved order parameter.

When the system is supposed to be closed, the average of the order parameter over the whole system (mean density) is conserved, but the existence of open boundaries through which the droplet phase evaporate once converted into the bulk phase leads to a decrease of this quantity. We are interested in the latter situation. We have previously studied the evaporation of periodic arrays of initially equal droplets in two-dimensional systems with open (absorbing) boundaries [9]. In the present work we extend the study by analyzing the evaporation of a set of initially randomly located identical droplets in a system with open boundaries.

One of the simplest models to describe heterogeneous systems showing phase coarsening is formulated through the Cahn-Hilliard equation $[4,6]$. In dimensionless form it reads

$$
\frac{\partial}{\partial t} c(\mathbf{r}, t)=\frac{D}{2} \nabla^{2}\left[2 c(1-2 c)(1-c)-\nabla^{2} c\right],
$$

where $D$ stands for the diffusion coefficient at the bulk phase. In what follows $D=1$. The two thermodynamically stable phases of our system correspond to different values of the order parameter $c: c \simeq 1$ for what is hereinafter referred to as the liquid (droplet) phase and $c \simeq 0$ corresponding to what will be called the vapor (bulk) phase; the third homogeneous solution $c=1 / 2$ is unstable. The surface tension associated with the interface between the liquid and the gas phase is denoted $\sigma$ in the following. It can be calculated as $\sigma=\int d x[d c(x) / d x]^{2}=\sqrt{2} / 6$, where $c(x)$ is a onedimensional solution of Eq. (1) satisfying the boundary conditions $\quad c(-\infty)=0$ and $c(\infty)=1$, i.e., $\quad c(x)=\frac{1}{2}[1$ $-\tanh (x / \sqrt{2})]$.

We numerically integrate Eq. (1) using an Euler discretization in a rectangular system of dimensions $L_{x}=256$ and $L_{y}=512$ with two absorbing boundaries $[c(t)=0]$ at $x=0$ and $x=L_{x}$ and periodic boundary conditions in the $y$ direction. The simulations start from a configuration of $N_{0}$ randomly located identical droplets. To prepare this initial configuration, we first integrate Eq. (1) with periodic boundary conditions in both directions and an initial condition generated by assigning to each point a concentration $c(r, 0)=c_{0}$ $+\eta(r), c_{0}$ being the mean concentration and $\eta$ a uniformly random number in the interval $[-0.05,0.05]$. Following Eq. (1), randomly located domains of droplets with $c \simeq 1$ form and grow in a bulk with $c \simeq 0$. After some time, when the system has $N_{0}$ droplets, we stop the simulation and enforce the droplets to have the same radius $R_{0}$. Then we use this configuration as the initial condition to study the effect of the absorbing boundaries.

Figure 1 shows some patterns corresponding to a typical evolution from an initial configuration of $N_{0} \approx 250$ droplets of equal radius $R_{0}=5.5$. We can observe how the droplets nearest each of the open boundaries start to evaporate soon, leaving a region without droplets whose thickness grow in 


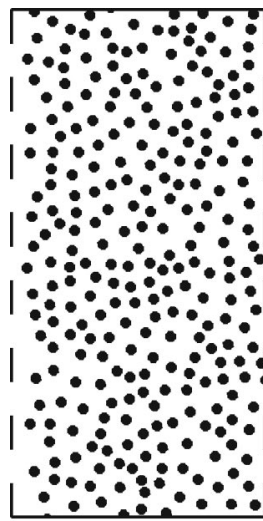

(a)

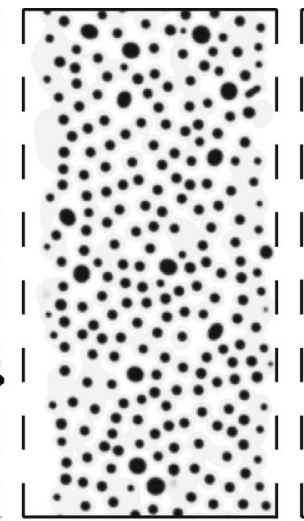

(b)

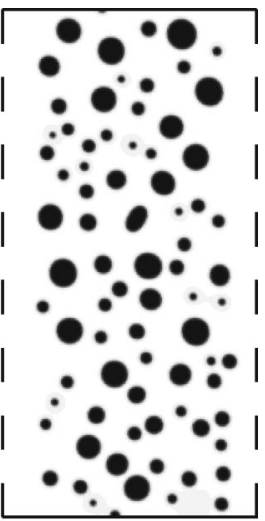

(c)
FIG. 1. Patterns showing the evolution of a droplet configuration, corresponding to an initial condition of $N_{0} \approx 250$ and $R_{0}$ $=5.5$ at times $t=0$ (a), 1000 (b), and 5000 (c). The absorbing boundaries in our open system are represented by discontinuous lines.

time. Far enough from the open boundaries, in the middle of the system, the phase separation process evolves like in a closed system. Between these two different regions there exists an intermediate zone that contains few droplets in the process of evaporation. In order to visualize better the evolution of the mean density, in Fig. 2 we plot the onedimensional concentration profile averaged along the $y$ direction $\bar{c}(x, t)=L_{y}^{-1} \int_{0}^{L_{y}} c(\mathbf{r}, t) d y$. This quantity is hereinafter denoted the density profile. In Fig. 3 we plot the time evolution of the whole number of droplets $N(t)$. This quantity shows a fast decrease at early and intermediate times and a slower behavior at longer times. The open boundaries favor the early disappearance of droplets that are near the boundary, in a faster process than for the same initial configuration but in a closed system. The evolution of the mean radius, measured as $\sqrt{2} R_{G}, R_{G}$ being the mean radius of gyration, is plotted in Fig. 4. It shows a long time behavior similar to the

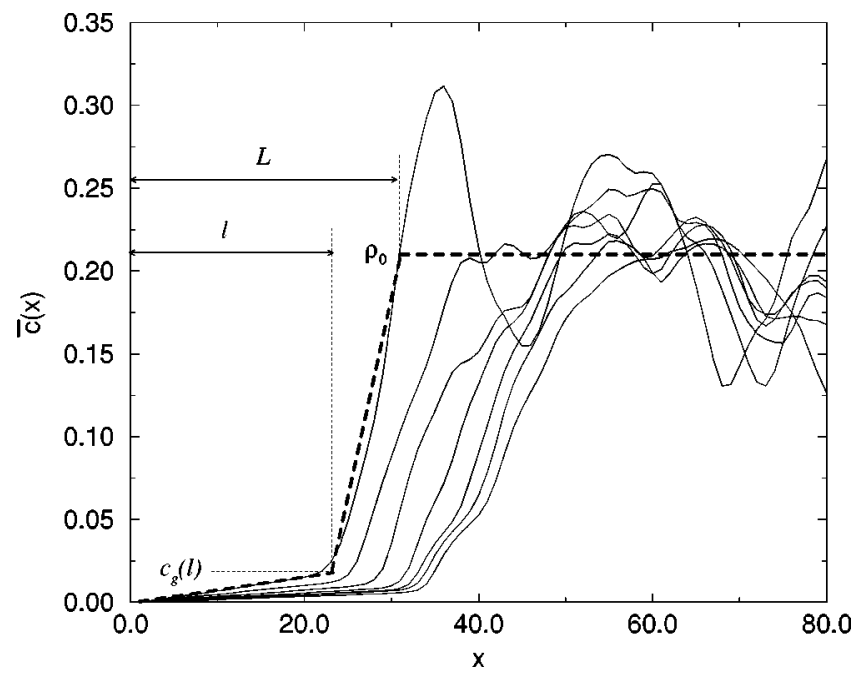

FIG. 2. Density profiles for the same conditions as in Fig. 1, corresponding to times from $t=2000$ to $t=14000$ every $\Delta t$ $=2000$. Dashed lines correspond to the model of the theoretical approach (see the text).

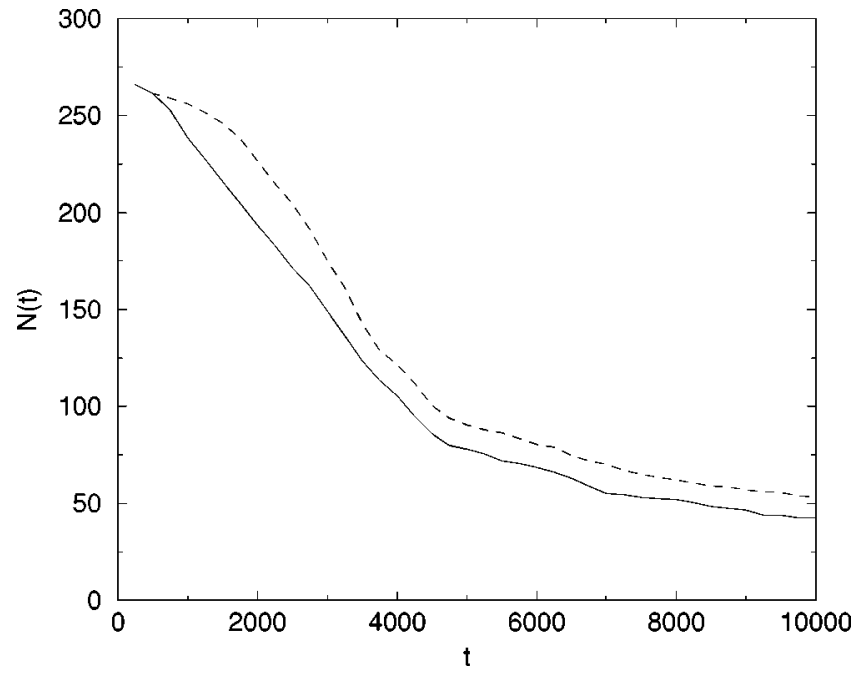

FIG. 3. Number of droplets versus $t$ for the same conditions as in Fig. 1 (solid line) and for a closed system with the same initial configuration (dashed line).

one obtained for a closed system. After some transient dominated by the initial condition and the open boundaries, the radius increases with the usual power law $t^{1 / 3}$, according with the Lifshitz-Slyosov theory.

We turn now to the theoretical discussion of the evaporation and coarsening dynamics described here. According to the density profiles shown above, after some very short transient, three different spatial regions can be distinguished in our system (see Fig. 2). The first one, whose width is denoted by $l(t)$, will hereinafter be referred to as a boundary layer region. It is the area nearest the absorbing walls and is quasihomogeneous (small linear profile) and purely composed of the leaving gas phase without any signature of liquid droplets. The second one is called a transition region and spatially extends from $l(t)$ to an abscissa hereinafter denoted $L(t)$ (measured from the open boundary). It is no longer homogeneous but rather contains a mixture of gas phase and liquid droplets. The important feature to be kept in mind for further discussion, however, is that those droplets, while they

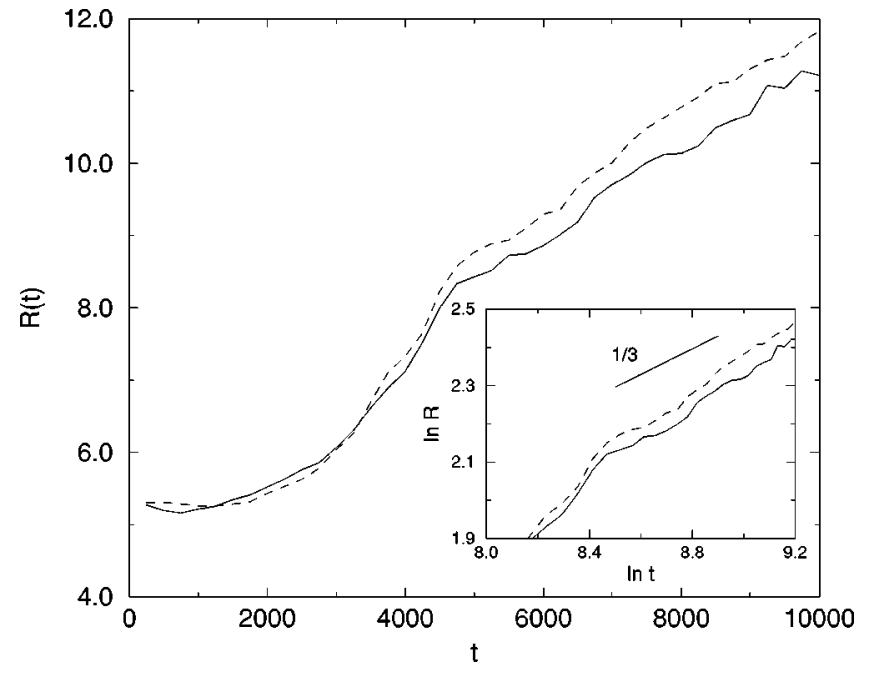

FIG. 4. Mean radius versus $t$ for the same conditions as in Fig. 1 (solid line) and for a closed system with the same initial configuration (dashed line). 
exist, do not move but irreversibly evaporate, feeding in this way the gas phase that will eventually escape from the system after crossing through the boundary layer. It is then clear that in this region the liquid droplets and the gas phase in their vicinity can never attain equilibration. Finally, the third region to be distinguished in the system is its bulk part. It is again a heterogeneous zone composed of liquid droplets, either growing or shrinking, and gas phase. In the following we will assume that the behavior in this region is completely autonomous with respect to the evaporation process of the system as a whole. In other words, we will assume that the bulk region keeps its mean density equal to the prescribed initial value for the entire distribution, denoted $\rho_{0}$, its behavior being purely that corresponding to a strictly conserved system. Thus all that happens in the bulk region is a typical coarsening process of the sort described by the standard Lifshitz-Slyosov theory for a closed system, as displayed by the dashed line in Fig. 4. Furthermore, contrarily to what was mentioned to occur in the transition region, we can properly invoke for the bulk region an equilibration principle between the existing droplets and the gas phase surrounding them. Taking into account surface tension effects (Young-Laplace corrections), this enables us to express the value of the order parameter in the bulk gas phase $c_{g}^{(b u l k)}$ in terms of the averaged radius of the droplets: $c_{g}^{(b u l k)}=\sigma / 2 R$.

The picture of the evaporation process that emerges from the previous considerations is then rather simple. The whole dynamics could be described as a replacement process of the bulk phase that is progressively invaded as time goes on by the boundary layer and the accompanying transition region, both propagating from the absorbing walls. Such a shrinkage of the bulk part of the system is naturally accompanied by a decrease in the overall density of the system since the "heavier" bulk region is being replaced by the "lighter" ones closer to the open boundaries. Actually, a material balance derived from this simple argument is all that we will invoke, as explained later on, to derive the basic equations for the relevant dynamical variables of our system. Needless to say, given the considerations above, we will simply need to in the following to the distinctive behavior of the evaporating boundary and transition layers since the bulk is going to be described by the standard Lifshitz-Slyosov theory.

Let us start by considering separately the gas phase of our heterogeneous system. In both the boundary and transition purely evaporating layers, the concentration of such a gas phase $c_{g}$ adopts very small values. This permits us to linearize the Cahn-Hilliard equation (1) leading, in the lowestorder approximation, to a pure diffusion equation for $c_{g}$,

$$
\frac{\partial}{\partial t} c_{g}(\mathbf{r}, t)=D \nabla^{2} c_{g}
$$

Furthermore, we will invoke a quasistatic approximation, legitimated due to the extremely slow evaporation dynamics here analyzed, that reduces the dynamics expressed by Eq. (2) to a pure Laplacian one. That is to say, a linear profile is going to be established for $c_{g}$ that extends from $c_{g}(0)=0$ at the absorbing boundary to the value well inside $c_{g}(L(t))$ $=c_{g}^{(b u l k)}$. In passing notice that this bulk value introduces a certain time dependence on this boundary condition through its dependence on the average radius of the bulk droplets $R(t)$. The full characterization of our model is still pending on the prescription of a boundary condition at the interface separating the boundary and transition layers. Lacking more fundamental arguments, we just assume as a sort of working hypothesis, to be checked later on, that $c_{g}(l(t))=\alpha c_{g}^{(b u l k)}$, in terms of an ad hoc introduced parameter $\alpha(\alpha<1)$ assumed to be time independent. In turn this will simply lead to a unique time scaling for the two spatial scales introduced so far in the system since within the Laplacian approximation invoked above $L(t)=l(t) / \alpha$.

Our next task will be to derive a dynamical equation for $l(t)$. As anticipated this will be accomplished in terms of a material balance formulated for an infinitesimal displacement of the interface separating the evaporating regions from the bulk one. Obviously this means that we are going to need to refer to the density profile of the mixed phase system rather than to the concentration of the gas phase. We have according to our description above,

$$
\begin{gathered}
\bar{c}(x, t)=\frac{c_{g}(l(t))}{l(t)} x, \quad 0 \leqslant x \leqslant l(t) \\
\bar{c}(x, t)=c_{g}(l(t))+\frac{\rho_{0}-c_{g}(l(t))}{L(t)-l(t)}[x-l(t)], \quad l(t) \leqslant x \leqslant L(t)
\end{gathered}
$$

$$
\bar{c}(x, t)=\rho_{0}, \quad x \geqslant L(t)
$$

where, according to our simulation results, we have supposed also a linear spatial distribution for the transition region (Fig. 2).

In terms of these density profiles, the differential mass evaporated from the system corresponding to an elementary displacement $d l(t)$ is readily calculated. After transforming it into a flux $j_{x}$ and since such a diffusive flux to the open boundary can also be trivially written as $j_{x}=-D[d \bar{c}(x, t) / d x]_{x=0}$, we finally end up with a differential equation for $l(t)$ given by

$$
\frac{d l^{2}}{d t}=\frac{4 D \alpha c_{g}^{(b u l k)}}{\left(1+\frac{1}{\alpha}\right) \rho_{0}-c_{g}^{(b u l k)}},
$$

where we have neglected the time dependence of $c_{g}^{(b u l k)}$.

A complementary equation for the spatially averaged density of matter in the system, denoted $\rho(t)$ is also readily calculated from the distributions above. Expressing it in terms of $l(t)$, we have

$$
\rho(t)=\rho_{0}-\frac{\left(1+\frac{1}{\alpha}\right) \rho_{0}-c_{g}^{(\text {bulk })}}{L_{x}} l(t) .
$$

Once combined, Eqs. (4) and (5) explicitly determine the temporal evolution of the averaged density of matter in the system 


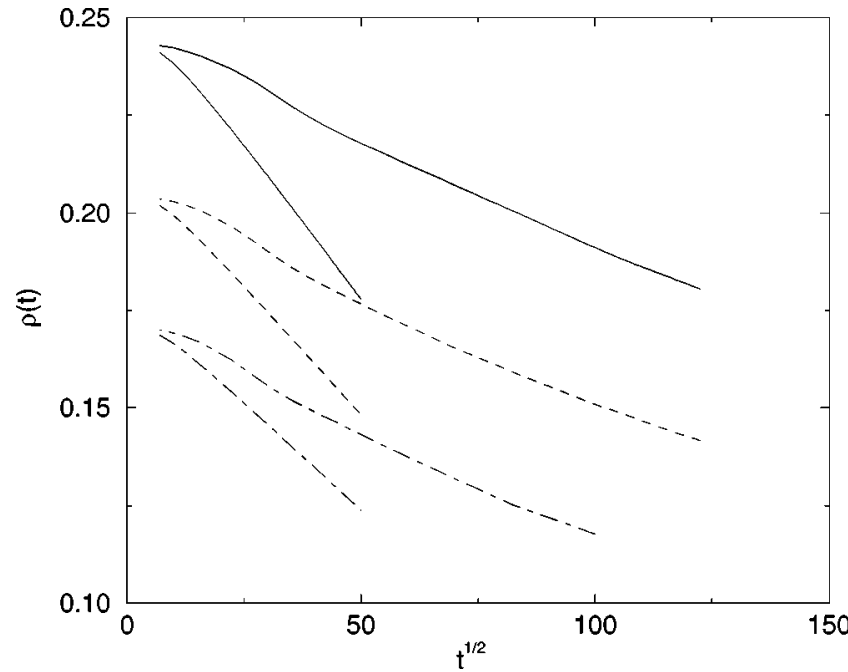

FIG. 5. Mean density versus $t^{1 / 2}$ for three configurations with $N_{0} \approx 250$ and different initial radii: $R_{0}=6$ (solid line), $R_{0}=5.5$ (dashed line), and $R_{0}=5$ (dot-dashed line). Free diffusion cases for the same initial conditions are also plotted for comparison (steepest lines).

$$
\frac{d\left(\rho_{0}-\rho\right)^{2}}{d t}=\frac{4 D}{L_{x}^{2}}\left[(\alpha+1) \rho_{0}-\alpha c_{g}^{(b u l k)}\right] c_{g}^{(\text {bulk })},
$$

whose solution reads explicitly

$$
\rho(t)=\rho_{0}-\frac{2}{L_{x}}\left\{\left[(\alpha+1) \rho_{0}-\alpha c_{g}^{(b u l k)}\right] c_{g}^{(b u l k)}\right\}^{1 / 2}(D t)^{1 / 2} .
$$

This is shown in Fig. 5, where we plot $\rho(t)$ versus $t^{1 / 2}$ for three droplets configurations corresponding to different initial radii. For time long enough it is well reproduced by linear fits. Actually, these enable us to indirectly evaluate the value of the parameter $\alpha$, which turns out to be $\alpha \approx 0.77$ for the three cases.

Let us compare these results with those that can be obtained for the free diffusion case, with the same diffusion coefficient,

$$
\frac{\partial}{\partial t} c(\mathbf{r}, t)=D \nabla^{2} c
$$

and with the same initial condition (droplets) and absorbing boundaries. What we observe in these parallel simulations is that the diffusion process homogenizes the center of the system to the mean density $\rho_{0}$ and the concentration evaporates through the boundaries but now in a faster dynamics than in the previous case. The analytical solution of this problem, with a homogeneous initial condition given by $\rho_{0}$, is straightforward [10] and the mean density decays as

$$
\rho(t)=\rho_{0}-\frac{2 \rho_{0}}{\pi^{1 / 2} L_{x}}(D t)^{1 / 2}
$$

A comparison of this result with Eq. (7) lead us to conclude that although both cases have the same mean density $\rho_{0}$ and the same diffusion coefficient $D$ in the bulk, in the coarsening case there is an "effective bulk density" now controlling the evaporations. This new quantity can be extracted by comparing Eqs. (7) and (9), thus obtaining

$$
\rho_{\text {eff }}=\left\{\pi\left[(\alpha+1) \rho_{0}-\alpha c_{g}^{(\text {bulk })}\right] c_{g}^{(\text {bulk })}\right\}^{1 / 2} .
$$

As $c_{g}^{(b u l k)} / \rho_{0} \ll 1$, the evaporation process is much slower when droplets are present. However the power decay law $t^{1 / 2}$ does not change.

Figure 5 summarizes the comparison just made. The presence of coarsening slows down drastically the evaporation dynamics, but qualitatively we still have a diffusive process, now controlled by the very small density of the bulk phase. Nevertheless, we should keep in mind that intrinsically the dynamics here analyzed is richer than that of a pure diffusive system. Actually, further refinements could be achieved by a numerical integration of Eq. (6) once $c_{g}^{(b u l k)}$ is expressed and evaluated in terms of the dynamically evolving average radius of the droplets. However, since the time evolution of this quantity is slower $\left(t^{1 / 3}\right)$ than the purely diffusive one, it is unlikely to expect significant quantitative differences with respect to the approximate analysis presented above.

From the above results we conclude that during the phase separation process (coarsening) in open systems, the evaporation of the liquid phase is controlled by diffusion mechanisms. Quantitatively one can say that we have a diffusion process controlled by the bulk "gas" density (smaller than the mean density) whose value is sustained by the dynamics of droplets. As the Lifshitz-Slyozov dynamics is much slower than a purely diffusive dynamics, the latter dominates, as it is observed in numerical simulations.

\section{ACKNOWLEDGMENTS}

This research was supported by an ACCIONES INTEGRADAS-DAAD grant, by DFG, by Dirección General de Investigación Científica y Técnica (Spain) under Project Nos. PB96-0241 and PB96-1001, and by the Comissionat per Universitats i Recerca de la Generalitat de Catalunya. We also acknowledge computing support from Fundació Catalana per a la Recerca.
[1] S. J. Langer, in Solids Far From Equilibrium, edited by C. Godreche (Cambridge University Press, Cambridge, 1992).

[2] J. D. Gunton, M. San Miguel, and P. Sahni, in Phase Transitions and Critical Phenomena, edited by C. Domb and J. L. Lebowitz (Academic, New York, 1983), Vol. 8.
[3] I. M. Lifshitz and V. V. Slyozov, J. Phys. Chem. Solids 19, 35 (1961).

[4] P. C. Hohenberg and B. I. Halperin, Rev. Mod. Phys. 49, 435 (1977).

[5] E. M. Lifshitz and L. P. Pitaevskii, Course on Theoretical 
Physics (Pergamon, Oxford, 1981), Vol. 10.

[6] J. W. Cahn and J. E. Hilliard, J. Chem. Phys. , 28, 258 (1958); 31, 688 (1959)

[7] A. M. Lacasta, A. Hernández-Machado, and J. M. Sancho, Phys. Rev. B 48, 9418 (1993).

[8] A. M. Lacasta, J. M. Sancho, and C. Yeung, Europhys. Lett.
27, 291 (1994).

[9] A. M. Lacasta, I. M. Sokolov, J. M. Sancho, and F. Sagués, Phys. Rev. E 57, 6198 (1998).

[10] A. Onuki, K. Sekimoto, and D. Jasnow, Prog. Theor. Phys. 74, 685 (1985). 